\title{
Description d'une nouvelle espèce de Dioctowittus (Nématode) et note sur la position systématique du genre
}

\author{
par Odile BAIN et Esmaïl GHADIRIAN
}

Chabaud et Le Van Hoa décrivaient en 1960 un Nématode Adénophoréen, Dioctowittus wittei, caractérisé par la régresssion très marquée de l'appareil sensoriel et la transformation de l'intestin en organe de réserve (trophosome). La forme des œufs et la présence d'une ventouse vestigiale sur la queue du mâle permettaient de le rapprocher de Dioctophyme renale; avec circonspection ces deux auteurs placèrent le genre dans la famille des Dioctophymatidae, mais en raison de ses très grandes particularités anatomiques, liées probablement à une vie tissulaire, ils l'isolèrent dans la nouvelle sousfamille des Dioctowittinae.

Aujourd'hui nous croyons pouvoir apporter des précisions sur les affinités du genre grâce à l'étude histologique de l'appareil digestif d'une nouvelle espèce découverte chez un serpent malgache.

\section{Description}

MATÉRIEL: Lot $1.005 \mathrm{~F}$; une femelle entière (holotype), des fragments antérieurs et postérieurs de femelle, et deux fragments antérieur et postérieur de mâle, récoltés dans la cavité générale de Lioheterodon modestus (Günther 1863) (Colubridae) à Ampijoroa (Madagascar), le 17-1-1962. Nous devons cet intéressant matériel à MM. Brygoo et Domergue et nous les en remercions très vivement.

Ce sont des Vers très longs; la bouche est allongée dorsoventralement ; les amphides sont réduites ; les papilles, très petites, sont à peine visibles ; il n'y a pas de capsule buccale; l'œsophage est nu dans la région antérieure et montre une lumière nette, à bords cuticulaires ; à ce niveau il est entouré par un manchon de petites fibres musculaires en fuseau, disposées parallèlement à l'axe du corps ; en arrière il est caché par des massifs très sombres, oblongs, disposés sur deux axes longitudinaux et irrégulièrement alternés (1) ; l'appareil excréteur et les diérides ne sont pas visibles; l'intestin est chargé de réserves et sa lumière n'est discernable que sur coupes épaisses.

(1) Chez D. wittei, contrairement à ce que pensaient Chabaud et Le Van Hoa, l'œsophage se continue de la même façon tout le long des massifs de réserves, atteignant ainsi une longueur de $19 \mathrm{~mm}$ chez la femelle et le mâle. 

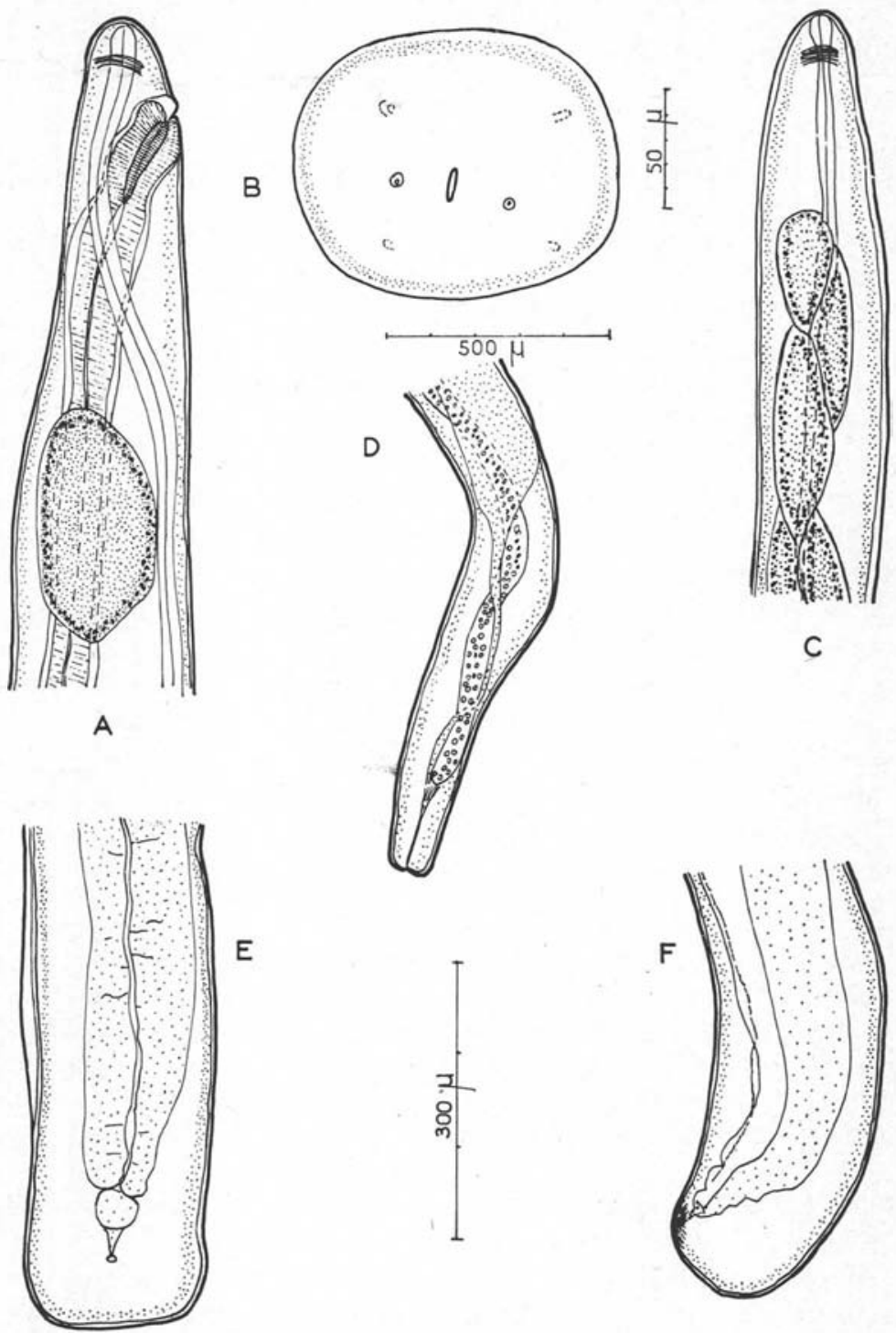

Fig. 1. - Dioctowittus chabaudi n. sp. Extrémités antérieures: A : femelle, vue latérale. B: femelle, vue apicale. C: mâle. Extrémités postérieures: D : femelle, vue latérale. E: mâle, vue ventrale. F : idem, vue latérale. A, C, D : échelle $500 \mu$. B : échelle $50 \mu$. E, F : échelle $300 \mu$

Femelle : Corps long de $234 \mathrm{~mm}$, large au maximum de $450 \mu$; anneau nerveux à $80 \mu$ de l'apex; œsophage long de $16 \mathrm{~mm}$; massifs œsophagiens débutant à $820 \mu$ de l'apex et larges de $200 \mu$ à $350 \mu$; l'anus n'est pas visible mais l'extrémité en cul-de- 
sac de l'intestin est retenue par un ligament dont le point d'attache sur la cuticule, souvent difficile à discerner, semble être terminal ; la vulve s'ouvre à $200 \mu$ de l'extrémité antérieure ; il n'y a qu'un tube génital ; l'utérus se termine à $18 \mathrm{~mm}$ de l'extrémité postérieure ; l'apex de l'ovaire situé à $180 \mu$ de la pointe caudale est attaché à la cuticule par l'intermédiaire d'un ligament; les œufs mûrs sont embryonnés; ils sont lenticulaires et mesurent $120 \mu$ de long, sur $70 \mu$ de large et $50 \mu$ d'épaisseur ; à leurs extrémités tronquées se trouve un bouchon muqueux autour duquel s'insère une touffe de filaments d'environ $200 \mu$ de longueur ; la queue est arrondie et dépourvue de formations cuticulaires.

Mâle : Longueur inconnue mais les fragments dont nous disposons atteignent environ $40 \mathrm{~mm}$; largeur maximum $300 \mu$; anneau nerveux à $70 \mu$ de l'apex ; œsophage long de $15 \mathrm{~mm}$; les massifs œsophagiens, larges de $110 \mu$ à $250 \mu$, naissent à $430 \mu$ de l'apex et se continuent sur une longueur d'environ $14,5 \mathrm{~mm}$; le testicule débute par une ampoule à parois assez épaisses, à environ $18 \mathrm{~mm}$ de l'apex ; orifice génital à $75 \mu$ de l'extrémité postérieure; spicule et gubernaculum absents; en avant du cloaque débutent de très fines ailes subventrales, longues de $2.350 \mu$; région caudale légèrement incurvée vers la face ventrale; queue arrondie, dépourvue de papilles et de ventouse vestigiale.

Discussion : La régression de l'appareil sensoriel, le tube génital unique, l'absence de spicule, la forme des œufs, l'absence d'anus et la présence des massifs œsophagiens permettent aisément d'identifier nos spécimens au genre Dioctowittus. Bien qu'ils soient très proches de $D$. wittei, ils s'en distinguent par quelques caractères : la séparation nette entre l'œsophage et l'intestin, la queue sans formations cuticulaires chez la femelle et sans ventouse vestigiale chez le mâle, les dimensions plus élevées des massifs œsophagiens, les filaments des œufs plus allongés. Nous pensons donc qu'il s'agit d'une nouvelle espèce, que nous appelons $D$. chabaudi n. sp.

\section{Données histologiques}

Pour mieux comprendre l'anatomie particulière de l'appareil digestif, trois régions du corps ont été étudiées chez les deux espèces : portion nue de l'œsophage, massifs œsophagiens et intestin. L'anatomie des deux espèces étant très voisine, la description effectuée sur $D$. chabaudi est généralement valable pour $D$. wittei; les différences de détail entre ces deux espèces sont signalées.

\section{Coupes transversales au niveau de la portion nue de l'asophage :}

Chez les deux espèces l'œsophage a une lumière de section triangulaire bien nette ; sa paroi, à surface interne cuticularisée, est constituée par un cytoplasme homogène, non glandulaire, traversé par des faisceaux plus ou moins nets de fibrilles contractiles ; il est entouré par une couche simple de petites fibres musculaires enveloppées chacune dans une mince couche de conjonctif ; ces fibres correspondent au « tissu scléreux » disposé en « mailles » dont parlaient Chabaud et Le Van Hoa. 


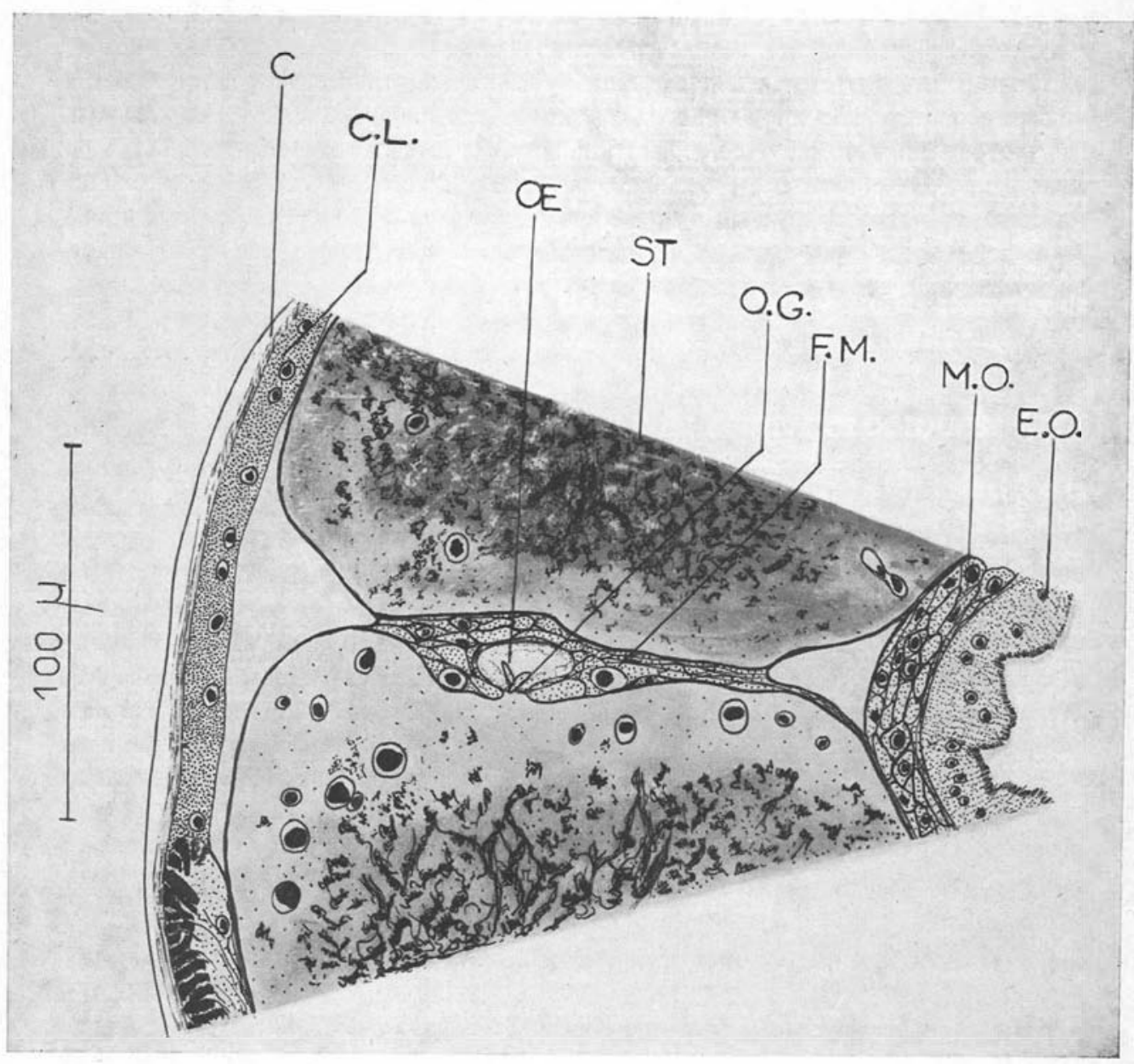

FIG. 2. - Coupe transversale de l'œsophage de D. chabaudi au niveau de l'orifice glandulaire d'un stichocyte (2 grains de sécrétion passent dans la lumière de l'œsophage)

\section{Coupes transversales au niveau des « massifs » asophagiens :}

Elles montrent que ces deux rangées de formations sont en réalité des glandes œsophagiennes hypertrophiées, les stichocytes, qui encadrent l'œsophage ; les deux stichosomes sont subventraux, accolés par leur face interne; chaque stichocyte a une structure syncitiale ; le cytoplasme est veiné, vacuolaire, acidophile et granuleux à la périphérie, très basophile au centre; les noyaux périphériques sont plus petits et plus nombreux que ceux du centre; chaque glande débouche dans la lumière de l'œsophage par un très petit orifice qui laisse passer quelques grains de sécrétion de la même 


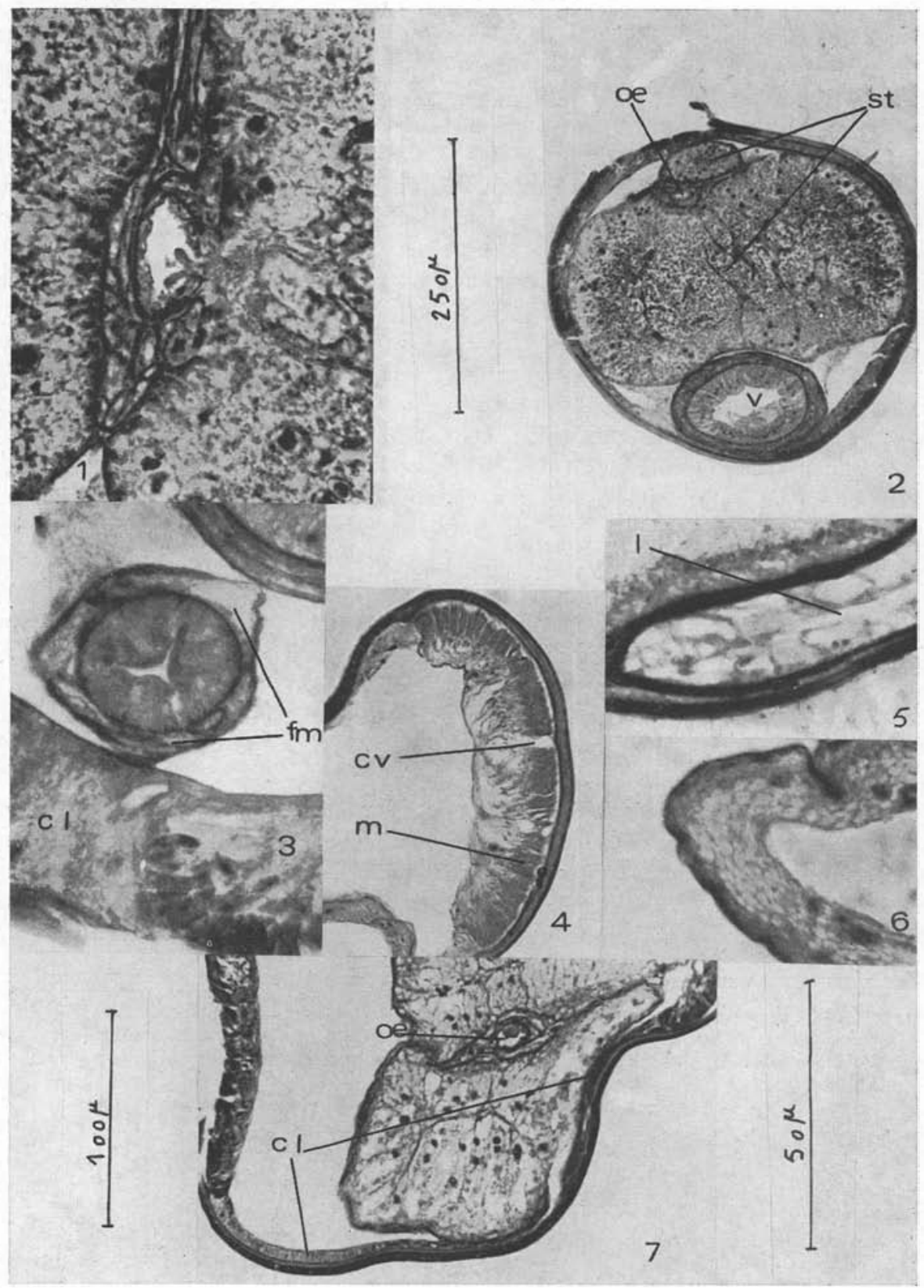


couleur que le cytoplasme périphérique. L'œsophage lui-même toujours tubuleux mais à lumière plus étroite est réduit à une mince couche cytoplasmique homogène, à noyaux très rares et à bord interne non cuticularisé ; il est entouré par les mêmes fibres que dans la région antérieure, mais celles-ci forment plusieurs couches.

\section{Coupes transversales au niveau de lintestin :}

Il est dépourvu de plateau absorbant ; sa lumière est réduite, peut-être même discontinue ; la paroi est formée par des mailles cytoplasmiques à noyaux rares, emprisonnant des gouttelettes lipidiques. Chez $D$. wittei la structure est la même mais les mailles cytoplasmiques ont une disposition régulière en écailles, et la lumière est plus grande.

En conclusion, l'appareil digestif de Dioctowittus, presque identique chez les deux espèces, est constitué par un œesophage très long et creux d'un bout à l'autre; les massifs œsophagiens ne sont pas des expansions du trophosome intestinal comme le supposaient Chabaud et Le Van Hoa, mais des stichocytes; seul l'intestin chargé de réserves constitue le trophosome.

Ces mêmes coupes nous ont permis accessoirement d'étudier le fourreau épidermomusculaire : il se caractérise par des cordes latérales très larges de structure syncitiale et riches en noyaux; dans la région antérieure, ces cordes sont épaisses, mais plus étroites, à cytoplasme très vacuolaire, surtout chez $D$. wittei, mais on n'observe pas de canalicules de sécrétion traversant la cuticule à leur niveau; les cordes latérales ne s'insinuent pas sous les cellules myoépithéliales. Les limites de celles-ci ne sont pas visibles; leurs sommets convergent vers les cordes ventrale ou dorsale.

\section{Discussion}

Malgré l'atrophie générale des organes, le genre Dioctowittus conserve un élément caractéristique : l'existence des stichosomes. Cet appareil glandulaire œsophagien ne se retrouve que chez deux groupes d'Adénophoréens : chez les Mermithoïdea et chez les Trichurö̈dea; il y a deux stichosomes chez les Mermithoïdea, les Trichuroïdea larvaires et les Cystoopsidae, tandis qu'il n'en persiste plus qu'un chez les Trichuroïdea

Pнотоs. - Coupes transversales de D. chabaudi, n. sp. et D. wittei Chabaud et Le Van Hoa 1960. 1: D. chabaudi, coupe au même niveau que la figure 2. 2 : idem, vue d'ensemble des 2 stichosomes; l'un des stichocytes est coupé au sommet, l'autre occupe presque toute la cavité du Nématode. 3: idem, structure de l'œsophage en avant des stichosomes. 4: D. wittei, champs musculaires latéraux-ventraux (coupe effectuée à la hauteur de l'intestin). $5: D$. chabaudi, détail de l'intestin. $6: D$. wittei, idem. 7: D. wittei, corde latérale, au niveau des stichosomes. 2: échelle $250 \mu ; 4$ et 7 : échelle $100 \mu ; 1,3,5$ et 6 : échelle $50 \mu$

C : cuticule

C.L. : corde latérale

C.V. : corde ventrale

E.O.: épithélium de l'ovéjecteur

F.M. : fibre musculaire périøesophagienne

$\mathrm{V}$ : ovéjecteur
Légende des abbréviations
L: lumière de l'intestin
M : champ musculaire
M.O. : musculature de l'ovéjecteur
OE : œsophage
O.G. : orifice glandulaire
St. : stichocyte 
adultes si l'on en excepte les Cystoopsidae. Au contraire chez les Dioctophymatoïdea, il n'y a pas de stichosome; l'appareil glandulaire est inclus dans l'œsophage et a conservé le type primitif des trois glandes œsophagiennes qui sont ici tubuleuses et ramifiées (cf. Roman, 1965).

L'existence des stichosomes chez Dioctowittus nous conduit donc à le retirer des Dioctophymatidae et à le rapprocher des Trichosyringina, c'est-à-dire des Mermithides et des Trichurides. Les deux stichosomes persistant chez les adultes et l'intestin transformé en trophosome évoquent les Mermithoïdea ; mais la biologie (parasite de Vertébrés et non d'Invertébrés) et l'appareil génital impair rapprochent Dioctowittus des Trichuroïdea et c'est dans cette superfamille que nous le plaçons.

Par la double rangée de stichocytes, les œufs embryonnés, et par un certain nombre de caractères, conséquence probable d'une adaptation à la vie tissulaire, tels que l'absence de bandes bacillaires et l'intestin en cul-de-sac, il présente plus particulièrement des ressemblances avec Cystoopsis accipenseri Wagner 1867, seul représentant de la famille Cystoopsidae Skrjabin 1923. Nous rassemblerons donc les deux genres dans cette famille. Peut-être cependant s'agit-il plus ici d'une convergence que de réelles affinités et nous préférons conserver deux sous-familles distinctes; ceci nous conduit à apporter quelques modifications à la définition des Cystoopsidae.

- Cystoopsidae Skrjabin 1923 : Trichuroïdea avec deux stichosomes chez l'adulte ; intestin terminé en cul-de-sac; pas de bandes bacillaires; spicule absent pouvant être remplacé par un pénis; vulve très antérieure; œufs embryonnés; parasites adaptés à la vie tissulaire.

- Cystoopsinae (Skrjabin 1923) : Cystoopsidae à stichocytes peu volumineux et unicellulaires ; intestin terminé dans une « vessie digestive »; femelle à corps divisé en deux régions, l'antérieure renfermant l'œsophage, le début de l'intestin et une partie du tube génital; tubes génitaux à nombreuses circonvolutions; chez le mâle, pénis enveloppé dans une gaine membraneuse; œufs « en citron ». Espèce type unique : Cystoopsis accipenseri Wagner 1867.

- Dioctowittinae Chabaud et Le Van Hoa 1960 : Cystoopsidae à stichocytes volumineux, à structure syncitiale ; intestin sans dilatation terminale ; corps non divisé ; tube génital peu sinueux; ni pénis, ni spicule ; œufs lenticulaires, portant des touffes de soies polaires. Espèce type : Dioctowittus wittei Chabaud et Le Van Hoa 1960. Autre espèce : $D$. chabaudi $\mathrm{n}$. sp.

\section{Résumé}

Dioctowittus chabaudi n. sp., provenant d'un Ophidien malgache, est décrit; il se caractérise principalement par l'absence de ventouse vestigiale chez le mâle et de formations cuticulaires caudales chez la femelle. Une étude histologique montre que les massifs œsophagiens correspondent à une double rangée de stichocytes. Ce caractère, joint à la morphologie du fourreau épidermo-musculaire, nous conduit à retirer ce genre des Dioctophymatoïdea et à le placer chez les Trichuroïdea, dans la famille des 
Cystoopsidae, avec deux sous-familles, toutes deux monogénériques, Cystoopsinae (Skrjabin 1923) et Dioctowittinae Chabaud et Le Van Hoa 1960.

\section{Bibliographie}

Chabaud (A. G.) et Le Van Hoa, 1960. - Nématodes parasites de Reptiles. Aphasmidiens. Parc Nat. Upemba. Mission G.F. de Witte, 65 (2), 59-64.

Roman (E.) in Grassé (P. P.), 1965. - Traité de Zoologie. Némathelminthes, 4 (2), 731 p. Edit. Masson et $\mathrm{C}^{1 \mathrm{e}}$, Paris.

(Laboratoire de Zoologie [Vers] [ $\mathrm{P}^{\mathrm{r}}$ A.-G. Chabaud], Muséum National d'Histoire Naturelle, 57, rue Cuvier, Paris, $5^{\circ}$ ) 